\title{
Comparative Analysis and Distribution of Classes of Bacteria in Diabetic Wound Infection Tertiary Care Hospital
}

\author{
B.S. Saravanan ${ }^{1}$, S. Swarupa Gnana Sudha Meriam² and Arbind Kumar Choudhary ${ }^{3 *}$ \\ ${ }^{1} \mathrm{GHQH}$, Erode, India \\ ${ }^{2}$ Department of Microbiology, ${ }^{3}$ Dept of Pharmacology, IRT-PMC, Erode, India \\ *Corresponding author
}

\begin{tabular}{|l|}
\hline Ke y w o r d s \\
$\begin{array}{l}\text { Wound healing, } \\
\text { Diabetic foot, Foot } \\
\text { ulcer, } \text { S. aureus }\end{array}$ \\
\hline Article Info \\
\hline $\begin{array}{l}\text { Accepted: } \\
\text { 04 May 2019 } \\
\text { Available Online: } \\
\text { 10 June } 2019\end{array}$ \\
\hline
\end{tabular}

A B S T R A C T 


\section{Introduction}

A wound is a type of injury in which the skin is torn, cut or punctured (open wound) or where blunt force trauma causes a contusion (closed wound). It is referred to as a sharp injury which damages the dermis of the skin. Also, wound is a breach in the skin and exposure of subcutaneous tissue following loss of skin integrity. It provides a moist, warm and fertile environment conductive to microbial colonization and proliferation. The wound contaminants may not persist but species that grow and divide may become established, causing colonization or infection. Infection in a wound delays healing and may result in wound breakdown or complete wound dehiscence ${ }^{1,2}$. Most of the bacterial species live on human skin, in the nasopharynx, gastrointestinal tract and other parts of the body with some potential of causing disease. Surgical operation, burns, trauma diseases and nutrition affect the body defences.

The skin barrier is disrupted by every skin incision and microbial contamination is unavoidable inspire of the best skin preparation. Wound infection results in prolonged hospital stay and increased trauma care and treatment cost. The severity of complications depends largely on the infecting pathogen and site of infection. Wound infection has been a source of worry in the field of medicine. Advances in control of infections have not totally eradicated this problem owing to development of drug resistance. ${ }^{2}$ Wounds can further be classified as accidental, pathological or post-operative according to its nature. Certain parasites (for example, Hook worm larvae) and bacteria (Treponema pallidum) can penetrate intact skin, but certain primary skin infections like impetigo is caused by Streptococcus pyogenes or S.aureus, or both gain access through abrasions, as minor traumatoskin is apart of everyday life. Infection of a wound is the successful invasion and proliferation by one or more species of microorganisms anywhere within the body's sterile tissues, sometimes resulting in pus formation. Development of wound infection depends on the interplay of many factors. The breaking of the host protective layer, the skin, and thus disturbing the protective functions of the layer, will induce many cell types into the wound to initiate host response.

Wound infections may occur following accidental trauma and injections, but postoperative wound infections in hospital are most common. Some infections are endogenous in which infection occurs from patient's own bacterial flora such as Staphylococcus aureus from skin and anterior nares or coliforms. Many infections are exogenous; skin and anterior nares are important sources of Staphylococci, spread of organisms from hospital staff and visitors occur by direct and indirect airborne routes. ${ }^{4,5}$ Wound infection has always been a major complication of surgery and trauma. In spite of modern standards of preoperative preparation, antibiotic prophylaxis and operative technique, postoperative wound infections remain a serious problem.

Surgical infections are the third most commonly reported nosocomial infections and they account for approximately a quarter of all nosocomial infections ${ }^{6}$. For any given type of operation, the development of a wound infection approximately doubles the cost of hospitalization. The factors which strongly predispose to wound infections include pre-existing illness, length of operation, wound class and wound contamination. The potential sources of postoperative infections are patient, hospital environment, food, other patients, staff, infected surgical instruments, dressings and even drugs and injections. The pathogens 
isolated from infections differ depending on the underlying problem, location and type of surgical procedure. Antibiotics are one of the most commonly prescribed drugs. ${ }^{7}$ Because of an overall rise in healthcare costs, lack of uniformity in drug prescribing and the emergence of antibiotic resistance, monitoring and control of antibiotic use are of growing concern and strict antibiotic policies should be warranted. Strict antibiotic prescribing policy significantly overcome the overuse of antibiotics and reduces the development of resistance to antibiotics. ${ }^{89}$ Prescription pattern analysis and culture sensitivity analysis will be more effective and help in making local policy for antibiotics prescription. ${ }^{10}$ Thus the present study deals with the antibiotic susceptibility pattern of the isolates which may be helpful in framing modified antibiotic policy. The aim of the study was to determine the bacteriology of wound infections and to study the antibiotic susceptibility pattern of the isolates. Following were the objectives of this study. To identify the bacteria, present in the sample isolated from the wound. To categorize the patients according to their causative organism, present in the infected site.

\section{Materials and Methods}

Prospective study was performed for a period of six months, from May 2015 to October 2015 The study was conducted in Government headquarters Hospital, Erode. Hospital ethical committees permission was obtained before stating the research. The study included patients with wound infections such as ulcer wounds, diabetic foot ulcer wounds, post operative wounds and was on antibiotic treatment. Design of data entry in a separate data entry form for incorporating patient details was designed. A separate consent form was designed to get consent from patient or his/her representative in order to take samples. The proposed prospective study was planned to be carried out for a period of six months.

\section{Results and Discussion}

Wound infection has always been a major complication of surgery and trauma. Antimicrobial resistance patterns are continually evolving and multidrug resistant organisms undergo progressive antimicrobial resistance, continuously updated data on antimicrobial susceptibility profile will continue to be essential to ensure the provision of safe and effective empiric therapies. The reasons for the differences in antimicrobial drug resistant pattern might be related to infection control practices or to timing of the introduction of the resistant organisms. The present study highlights to determine the bacteriology of wound infections and the antibiotic susceptibility pattern of the organism isolated. ${ }^{11,12}$

A total of 120 samples were collected from patients with clinical evidence of wound infection (patients with complaints of discharge, pain, swelling, foul smelling and chronic wound) from January to July, 2015. Out of 120 samples collected, 20 samples were excluded from the study for the reason of negative culture. National mastitis council reports indicate that $25-40$ per cent of all clinical samples are negative on routine culturing. The possible reasons include, that the organism may no longer present and the clinical signs are due to by-products such as endotoxins. Another reason may be the antibiotics have killed the organism or suppressed organism numbers to unrecoverable levels. The incidence of wound infection was more common in males $(63 \%)$ than in females. (37\%) (Fig. 1). This might be explained by the fact that patient related factors like smoking, pre-existing infection may lead to colonization with $S$. aureus. This finding can be compared to the gender 
distribution given by Ohalete et al. Out of 100 culture positive samples, 61 samples were from diabetic ulcer patients, 20 samples were from ulcer patients, and 19 samples were from post-operative patients (Fig. 2). From the results, the most prevalent infection site was found to be diabetic foot ulcer. Diabetic foot ulcer wounds are increasingly common problem in people with diabetes and now constitute the most frequent diabetes related cause of hospitalization. People with diabetes have about a $25 \%$ chance of developing a foot ulcer in their lifetime, about half of which are clinically infected at presentation. Their prevalence and severity are largely a consequence of host-related disturbances (immunopathy, neuropathy and arteriopathy) and secondarily, pathogen-related factors (virulence, antibiotic resistance and microbial load). Our study complies with the findings in the study conducted by Anne et al. In the prospective data, surgical site infections are less common when compared with diabetic ulcer foot cases. The reason may be surgical site infections are preventable in most cases by following evidence-based guidelines for hand hygiene, administration of prophylactic antibiotics, and preoperative patient temperature management. Of the 100 samples (culture positive), $71 \%$ of culture positive wounds showed mono-microbial growth, $29 \%$ showed two type of microbial growth (Table 3) Similarly high percentage $(91.6 \%)$ of mono-microbial growth was reported by Mama et $\mathrm{al}^{13}$.

Out of 61 wound samples from diabetic ulcer sites 47 samples were yielded one type of organism while 14 samples were yielded two types of organism growth (Table 1 and 2 respectively). Number of cases with more than one organism is found to be more in diabetic ulcer cases when comparing with ulcer cases and surgical site cases. As the wound deteriorates deeper structures are affected. Anaerobes become more common, oftentimes infections show multiple growths of organisms. ${ }^{14}$ This finding comply with the results in the study conducted by Anne et al. The most frequently isolated organisms from diabetic foot ulcer sites were $S$. aureus (49.18\%), followed by E. coli (29.50\%), Proteus (21.31\%), Pseudomonas aeruginosa $(14.75 \%)$ and Klebsiella $(8.19 \%)$. This is in agreement with Mohammed et al., ${ }^{15}$ who reported that $S$. aureus is the most prevalent organism in wound site, accounting for almost $60 \%$ of the isolates. Out of the 20 wound samples Isolated from ulcer sites 15 samples yielded one type of organism and 5 samples were yielded two types of organisms (Table 3 and 4 respectively). The most frequently isolated organisms from ulcer sites were $S$. aureus (60\%), followed by Proteus (30\%), Klebsiella and E. coli (15\%).

The second most prevalent organism isolated was Proteus. The microbial flora in wound change over time, in early acute wound normal skin flora predominates. After about 4 weeks facultative anaerobic gram-negative rods will colonize the wound. ${ }^{16}$ Klebsiella and E. coli showed least percentage of distribution patterns $(15 \%)$ in ulcer wound site. The reason may be that wounds with a sufficiently hypoxic and reduced environment are susceptible to colonization by $E$. coli and Klebsiella ${ }^{17}$ Klebsiella bacteria are normally found in human intestines (where they do not cause any infection). In health care settings, Klebsiella infections commonly occur among sick patients who are receiving treatment for other conditions and who are taking long course of certain antibiotics are most at risk for Klebsiella infection In hospital settings, Klebsiella can be spread through person to person contact and are not spread through air. In this study absence of Pseudomonas was identified in ulcer site. This may be due to organisms like Pseudomonas are not very invasive unless the patient is highly compromised. Prevalence of S. aureus in the 
prospective data is in agreement with Nkang 39 et al. Out of 19 wound cases from surgical site 17 samples were with one type organism and 2 samples were with two types of organisms (Table 8 and 9 respectively) (Fig. $3-6)$.

Figure.1 Gender wise distribution of patients with wound infections

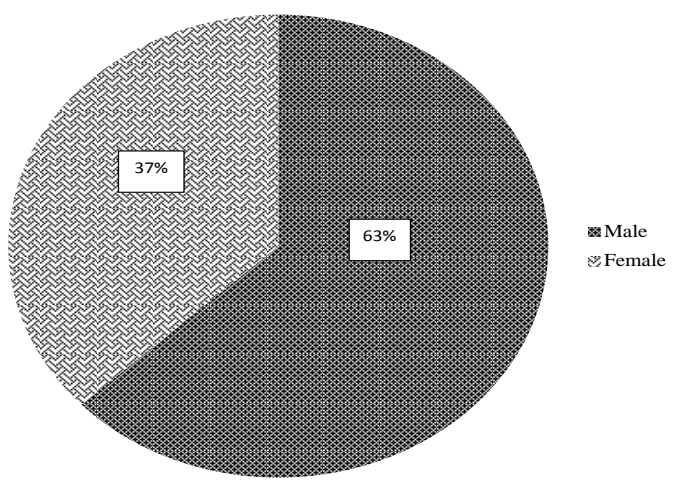

Types of wound infections

Figure.2 Percentage distributions of isolates from different wound sites of patients

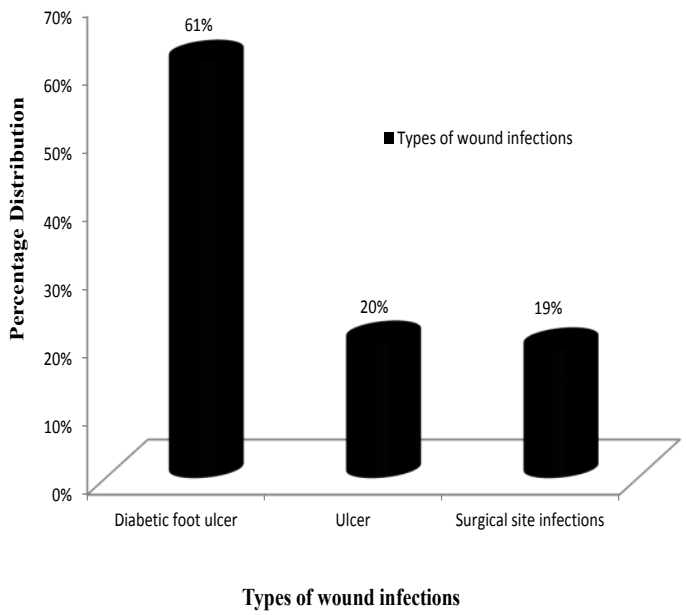


Microbiology of samples from diabetic foot ulcer $(n=61)$

Figure.3 Organisms isolated from infection site $(\mathrm{n}=100)$

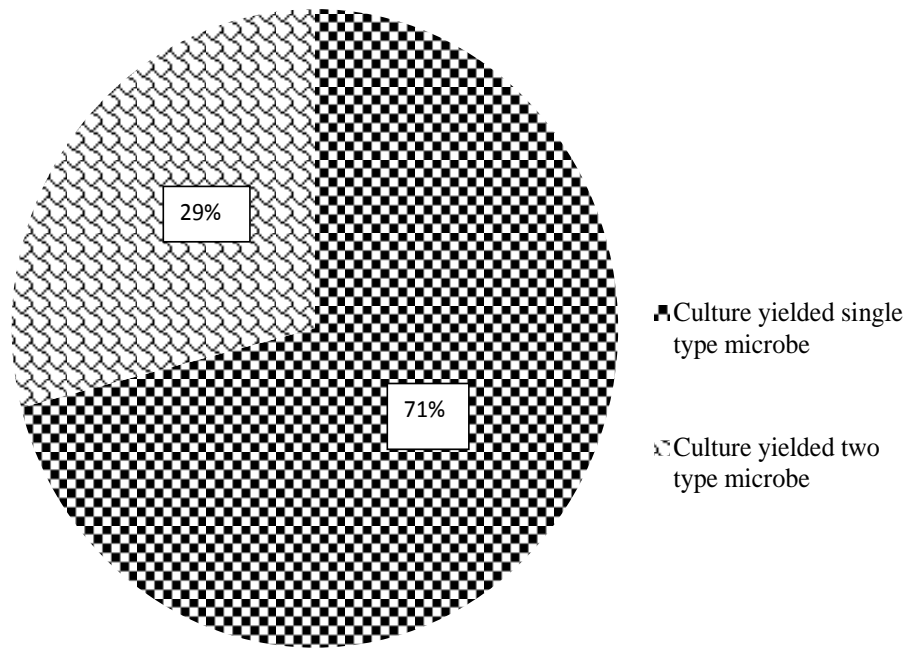

Table.1 Culture that yielded single type organism $(n=47)$

\begin{tabular}{|c|c|c|}
\hline 1 & S.aureus & 18 \\
\hline 2 & Proteus & 10 \\
\hline 3 & E.coli & 11 \\
\hline 4 & Pseudomonas aeruginosa & 5 \\
\hline 5 & Klebsiella & 3 \\
\hline
\end{tabular}

Table.2 Culture that yielded two type of organisms $(n=14)$

\begin{tabular}{|l|l|c|}
\hline Sl.No & \multicolumn{1}{|c|}{ Organism } & $\begin{array}{c}\text { Jumber of cases } \\
(\mathbf{n = 1 4})\end{array}$ \\
\hline $\mathbf{1}$ & E.coli+S.aureus & $\mathbf{4}$ \\
\hline $\mathbf{2}$ & S.aureus +Pseudomonas & $\mathbf{3}$ \\
\hline $\mathbf{3}$ & S.aureus+Proteus & $\mathbf{2}$ \\
\hline $\mathbf{4}$ & S.aureus+Klebsiella & $\mathbf{2}$ \\
\hline $\mathbf{5}$ & E.coli+Pseudomonas & $\mathbf{1}$ \\
\hline $\mathbf{6}$ & E.coli+Proteus & $\mathbf{1}$ \\
\hline $\mathbf{7}$ & S.aureus + E.coli & $\mathbf{1}$ \\
\hline
\end{tabular}


Microbiology of samples from ulcer site $(n=20)$

Figure.4 Percentage distribution of bacteria isolate from diabetic foot ulcer sites

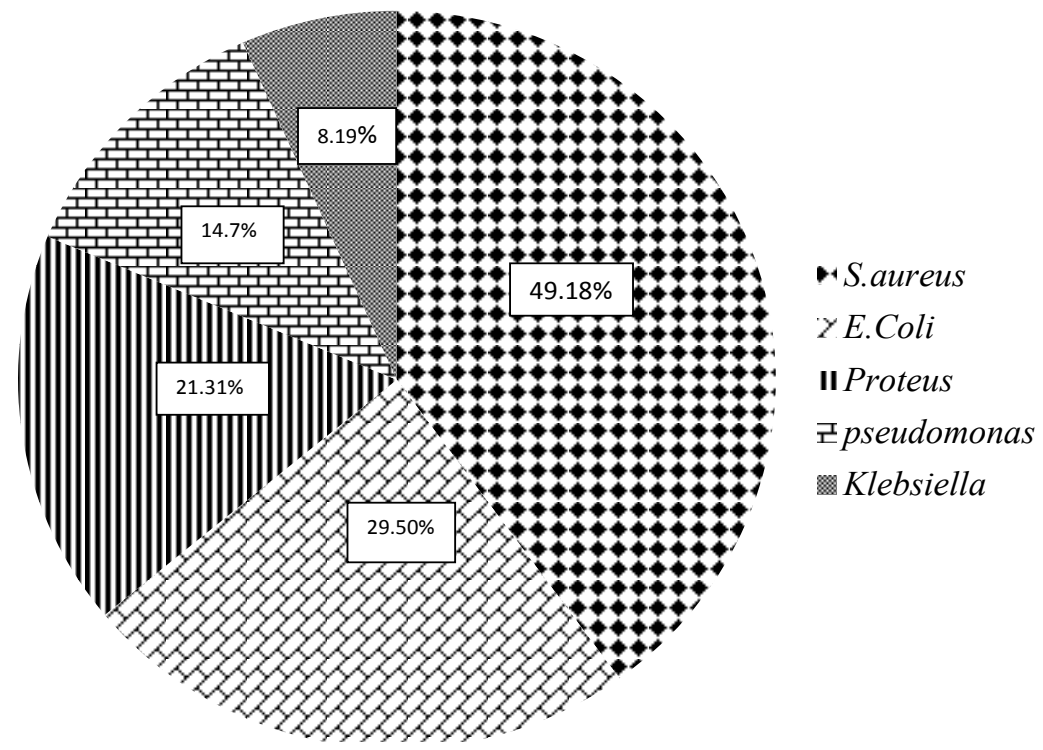

Table.3 Culture that yielded one type of organism $(n=15)$

\begin{tabular}{|c|c|c|}
\hline Sl.No & Organisms & $\begin{array}{c}\text { Number of cases } \\
(\mathbf{n}=15)\end{array}$ \\
\hline $\mathbf{1}$ & S.aureus & $\mathbf{8}$ \\
\hline $\mathbf{2}$ & Proteus & $\mathbf{3}$ \\
\hline $\mathbf{3}$ & E.coli & \\
\hline $\mathbf{4}$ & Klebsiella & $\mathbf{2}$ \\
\hline
\end{tabular}

Table.4 Culture that yielded two types of organisms $(n=5)$

\begin{tabular}{|c|c|c|}
\hline Sl.No & Organism & $\begin{array}{c}\text { Numberof } \\
\text { cases } \\
(\mathbf{n = 1 7})\end{array}$ \\
\hline $\mathbf{1}$ & S.aureus & $\mathbf{1 3}$ \\
\hline $\mathbf{2}$ & Pseudomonas & $\mathbf{3}$ \\
\hline $\mathbf{3}$ & E.coli & $\mathbf{1}$ \\
\hline
\end{tabular}


Table.5 Culture that yielded single type organism $(n=17)$

\begin{tabular}{|c|c|c|}
\hline Sl.No & Organism & $\begin{array}{c}\text { Number of } \\
\text { cases } \\
(\mathbf{n}=\mathbf{5})\end{array}$ \\
\hline $\mathbf{1}$ & S.aureus+Proteus & $\mathbf{2}$ \\
\hline $\mathbf{2}$ & Proteus+Klebsiella & $\mathbf{1}$ \\
\hline $\mathbf{3}$ & S.aureus + Klebsiella & $\mathbf{1}$ \\
\hline $\mathbf{4}$ & S.aureus + E.coli & $\mathbf{1}$ \\
\hline
\end{tabular}

Figure.5 Percentage distribution of bacteria isolates from surgical sites

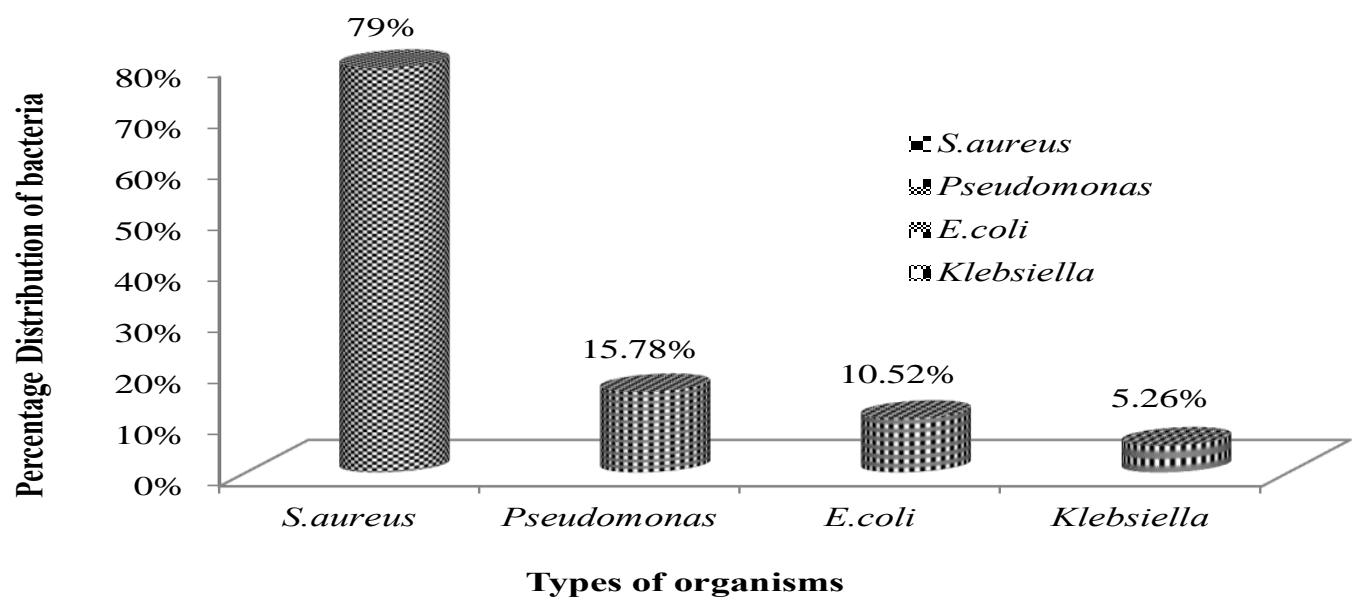

Microbiology of samples from surgical site infections $(n=19)$

Table.6 Culture that yielded two type organism $(n=2)$

\begin{tabular}{|c|c|c|}
\hline SI.No & Organism & $\begin{array}{c}\text { Number } \\
\text { of cases } \\
(\mathbf{n = 2})\end{array}$ \\
\hline $\mathbf{1}$ & S.aureus+Klebsiella & $\mathbf{1}$ \\
\hline $\mathbf{2}$ & S.aureus+E.coli & 1 \\
\hline
\end{tabular}


Figure.6 Percentage distribution of bacteria isolates from surgical sites

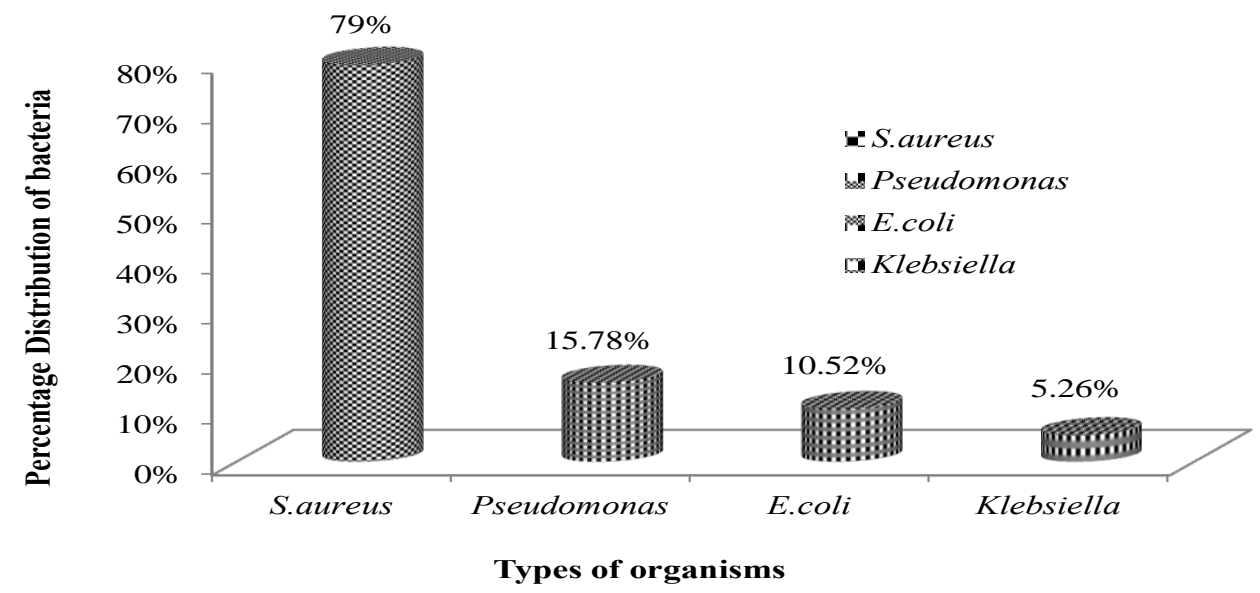

Table.7 Total number of organisms isolated from different wound infections $(n=100)$

\begin{tabular}{|c|c|c|}
\hline Sl.No & Organisms & $\begin{array}{c}\text { Number of cases collected } \\
(\mathbf{n = 1 0 0})\end{array}$ \\
\hline $\mathbf{1}$ & S.aureus & $\mathbf{5 7}$ \\
\hline $\mathbf{2}$ & E.coli & $\mathbf{2 3}$ \\
\hline $\mathbf{3}$ & Proteus & $\mathbf{1 9}$ \\
\hline $\mathbf{4}$ & Pseudomonas & $\mathbf{1 2}$ \\
\hline $\mathbf{5}$ & Klebsiella & $\mathbf{8}$ \\
\hline
\end{tabular}

The most frequently isolated organisms from surgical sites were $S$. aureus (78.94\%), Followed by Pseudomonas ${ }^{18}$, E. coli $(10.52 \%)$, and Klebsiella $(5.26 \%)$. Surgical wounds will heal rapidly if blood perfusion is maximized, thus delivering oxygen, nutrients, and cells of the immune system to the site of injury and providing minimal opportunity for microorganisms to colonize and proliferate. ${ }^{19}$

The pathogens isolated from infections differ, primarily depending on the type of surgical procedure. In clean surgical procedures, in which the gastrointestinal, respiratory tracts and gynecologic have not been entered, $S$. aureus from the exogenous environment or the patients skin flora is the usual cause of infection. In other categories of surgical procedures, including clean- contaminated, contaminated and dirty, the multiple growth of aerobic and anaerobic flora closely resembling the normal endogenous microflora of the surgically resected organ are the most frequently isolated pathogens. Our findings resembles with the result of Eriksen et al., in case of prevalence of isolated pathogens ${ }^{20}$.

The most prevalent organism isolated from different wound infections was found to be $S$. aureus $(57 \%)$, followed by E. coli (23\%), Proteus (19\%), Pseudomonas (12\%) and Klebsiella (8\%). (Table 10) Most prevalent organism isolated from different wound site was found to be $S$. aureus. This is not unexpected since the organism is a commensal or normal flora on the skin. One 
of the reasons that $S$. aureus is a frequent cause of infections is that it can survive for months on any type of surface. S. aureus cells also possess a wide armamentarium of virulence factors. ${ }^{21}$ These virulence factors include factors for adherence, for cell internalization, for evasion of host defense mechanisms, and for invasion of host tissue. With the help of these virulence factors, $S$. aureus is able to colonize the skin and mucous membranes of more than $30 \%$ of the human population.

In conclusion, this study gives us an insight to the current state of causative pathogens and. their sensitivity to different antibiotics used in Government headquarters. hospital, Erode. The prevalence of S. aureus from different wound infections in Erode government headquarters hospital was found to be high. High level of sensitivity was observed to Amikacin, Imipenem and Piperacillin Tazobactum. Likewise, high level of resistance was observed to Cephalosporins and Penicillin derivatives.

The data of this study may be used to determine trends in antimicrobial susceptibilities, to modify antibiotic policy and overall to assist clinicians in the rational choice of antibiotic therapy to prevent misuse, or overuse, of antibiotics in Government headquarters hospital, Erode. We suggest taking local infecting organism /sensitivity pattern into account when formulating prophylaxis as well as empirical therapy guideline for individual wound infection. We also suggest that the chosen antibiotic must have Antimicrobial susceptibility for the common prevalent stains of micro-organisms. The reasons for the differences in antimicrobial drug-resistant patterns might be related to infection control practices or to timing of the introduction of resistant organisms. However, more research is needed to clarify these differences.

\section{References}

1. Uwaezuoke JC, Nnodim JK. Bacteriology of Different Wound Infections and Their Antimicrobial Susceptibility Patterns in Owerri. Journal of Progressive Research in Biology. 2015; 1(1): 67- 69.

2. Mohammed A, Adeshina G, Ibrahim Y. Retrospective incidence of wound infections and antibiotic sensitivity pattern: a study conducted at the Aminu Kano Teaching Hospital, Kano, Nigeria. International Journal of Medicine and Medical Sciences. 2013; 5(2): 60 - 66.

3. Ramesh R, Sumathi S, Anuradha K, Venkatesh D, Krishna S. Bacteriology of postoperative wound infections. International Journal of Pharmaceutical and Biomedical Research. 2013; 4(2): 72-76

4. Walter CJ, Dumville JC, Sharp CA, Page T. Systematic review and meta-analysis of wound dressings in the prevention of surgical-site infections in surgical wounds healing by primary intention. British journal of surgery. 2012; 99(9): 1185-94.

5. Anderson DJ, Sexton DJ. Epidemiology and pathogenesis of and risk factors for surgicalsite infection. UpToDate. 2008. http://www. uptodate.com.

6. Mangram AJ, Horan TC, Pearson ML, Silver LC, Jarvis WR. Guideline for prevention of surgical site infection: 1999. Sciencedirect. 1999; 27(2): 97-134.

7. Syed AA, Tahir SM, Abdul SM, Noshad AS. Pattern of pathogens and their sensitivity isolated from superficial surgical site infections in a tertiary care hospital. Journal of Ayub Medical College Abbottabad. 2009; 21(2): 80 -82.

8. Klevens RM, Edwards JR, Richards CL. Estimating health care associated infections and deaths in U.S hospitals. Public health reports. 2007; 122(2): 160-66.

9. Cruse PJ, Foord R. The epidemiology of wound infection. Surgical clinics of North America. 1980; 60(1): 27-40.

10. Rosenthal VD, Richtmann R, Singh S. Surgical site infections, International 
nosocomial infection control consortium report. Infection control of hospital epidemiology. 2013; 34(6): 597-604.

11. Girish MB, Kumar TN. Culture and sensitivity pattern of micro-organism isolated from diabetic foot infections in a tertiary care hospital. International journal of current biomedical and pharmaceutical research. 2011; 1(2): $34-40$.

12. Redkar R, Kalns J, Butler W, et al., Identification of bacteria from a nonhealing diabetic foot wound by $16 \mathrm{~S}$ rDNA sequencing. Molecular and Cellular Probes. 2000; 14(3): 163-169.

13. Mama M, Abdissa A, Sewunet T. Antimicrobial susceptibility pattern of bacterial isolates from wound infection and their sensitivity to alternative topical agents at Jimma University Specialized Hospital, South-West Ethiopia. Annals of Clinical Microbiology and Antimicrobials. 2014; 13(5): 1-10.

14. Ohalete CN, Obi RK, Emea MC: Bacteriology of different wound infection and their antimicrobial susceptibility patterns in Imo state Nigeria. World Journal of Pharmacy and Pharmaceutical Science. 2012; 1(3): 1155-1172

15. Abid M, Adeshina GO, Ibrahim YKE. Retrospective incidence of wound infections and antibiotic sensitivity pattern: A study conducted at the Aminu Kano Teaching Hospital, Kano, Nigeria. International Journal of Medicine and Medical Sciences. 2013; 5(2): 60- 66.
16. Klebsiella pneumonia in healthcare settings. Www.cdc.gov/HAI/organisms/ Klebsiella/Klebsiella.html. Updated on 27thaugust 2012. Assessed on 2ndaugust 2015.

17. Pseudomonas aeruginosa in healthcare settings. Www.cdc.gov/HAI/organisms/ Pseudomonas/Pseudomonas.html. Updated on 7thmay 2014. Assessed on 27thmay 2014.

18. Arulmoli SK, Sivachandiran S, Perera BJC. Prescribing patterns of antibiotics for children before admission to a paediatric ward in Jaffna Teaching Hospital. Sri Lanka Journal of Child Health. 2009; 38(4): 121- 123.

19. Palikhe N. Prescribing Pattern of Antibiotics in Paediatric Hospital of Kathmandu Valley. Journal of Nepal Health Research Council. 2004; 2(2): 3136.

20. Tsai SS, Huang JC, Chen ST, et al. Characteristics of Klebsiella pneumonia Bacteremia in Community-acquired and Nosocomial Infections in Diabetic Patients. Chang gung Medical Journal. 2010; 33(5); 532-539.

21. Podschun R and Ullmann U. Klebsiella spp. as Nosocomial Pathogens: Epidemiology, Taxonomy, Typing Methods, and Pathogenicity Factors. Cinical Microbiological Review. 1998; 11(4): 589-603.

\section{How to cite this article:}

Saravanan, B.S., S. Swarupa Gnana Sudha Meriam and Arbind Kumar Choudhary. 2019. Comparative Analysis and Distribution of Classes of Bacteria in Diabetic Wound Infection Tertiary Care Hospital. Int.J.Curr.Microbiol.App.Sci. 8(06): 70-80. doi: https://doi.org/10.20546/ijcmas.2019.806.010 